\title{
Zehn Jahre Kinder- und Jugendpsychiatrie in Österreich: ein neues ärztliches Sonderfach in den Strukturen des Gesundheitswesens
}

\author{
Charlotte Hartl · Andreas Karwautz (D)
}

Online publiziert: 30. August 2017

(C) Der/die Autor(en) 2017. Dieser Artikel ist eine Open-Access-Publikation.

\begin{abstract}
Zusammenfassung Wir besprechen die kooperative Arbeit, die für die Entwicklung der Kinder- und Jugendpsychiatrie in Österreich geleistet wurde, fassen den Status-Quo der Versorgung in verschiedenen Settings zusammen und stellen weitere Entwicklungen dar. Für die intramurale Versorgung stehen derzeit etwa $50 \%$ der Plätze zur Verfügung, wobei sich die Verteilung über das Land als sehr heterogen erweist. Die extramurale Versorgung ist derzeit zu etwa $25 \%$ ausgebaut. Wir berechneten einen extramuralen Vollausbau mit dem Jahr 2033. Die weitere Entwicklung des Faches Kinder- und Jugendpsychiatrie wird nur mit gemeinsamen Anstrengungen aller Verantwortlichen gelingen.
\end{abstract}

Schlüsselwörter Versorgung · Austria $\cdot$ Kinder- und Jugendpsychiatrie $\cdot$ Ärzteausbildung

Ten years of child and adolescent psychiatry in Austria: a new medical speciality within the structures of public health services

Summary We discuss the comprehensive work for the development of child and adolescent psychiatry in Austria, summarize the current status of care in various settings and focus on further developments. Intramural care offers about $50 \%$ of the places needed and is heterogeneously distributed over the country, extra-

\footnotetext{
Dr. C. Hartl, MAS

Kassenordination für Kinder- und Jugendpsychiatrie,

Hiessbergergasse 2/5/2, Purkersdorf, Österreich

charlotte.hartl@medway.at

Univ.-Prof. Dr. A. Karwautz, FAED ( $\bowtie)$

Universitätsklinik für Kinder- und Jugendpsychiatrie,

Medizinische Universität Wien, General Hospital of Vienna,

Währinger Gürtel 18-20, 1090 Wien, Österreich

andreas.karwautz@meduniwien.ac.at
}

mural care offers already around one quarter of care in need. We calculated a fully developed extramural care system from about 2033. Further development of the Austrian care system in child and adolescent psychiatry needs collaborative efforts of all responsible players.

Keywords Care · Austria · Child and Adolescent Psychiatry $\cdot$ Training

\section{Einleitung}

Dieser Artikel referiert die verschränkte politische Arbeit der wissenschaftlichen Fachgesellschaft „Österreichische Gesellschaft für Kinder- und Jugendpsychiatrie, Psychosomatik und Psychotherapie - ÖGKJP“ und der Kinder- und Jugendpsychiatrie (KJP) und der Fachgruppenvertretung KJP in der Österreichischen Ärztekammer (ÖAK). Das ärztliche Handeln nach Leitlinien, gefordert in unserer täglichen Arbeit als versorgende ÄrztInnen, und der Ressourcenmangel eines neu etablierten Faches, stellen für alle KollegInnen ein Spannungsfeld dar. Die politischen Funktionen eröffnen Räume, praktische Erfahrungen auf direktem Weg einzubringen, und das Fach in seinen Aufgaben voranzubringen. Die Freude am Gestalten gibt Kraft, für nächste Generationen einen Beitrag zu leisten.

Die letzten 10 Jahre ermöglichten der ÖGKJP und der ÖÄK sowohl eine Weiterentwicklung der fachärztlichen Ausbildung und Kompetenz, als auch eine Implementierung von neuen KJP-Versorgungsstrukturen in das bestehende komplexe öffentliche österreichische Gesundheitssystem (vgl. zum Stand 2007: [1]), vgl. zur Situation in Deutschland: [2].

In der Facharztausbildung wurde die psychotherapeutische Dimension als Teil des Rasterzeugnisses implementiert, und mit der Vermehrung von Ausbildungsplätzen haben alle verantwortlich Beteiligten 
auf den Fachärztemangel reagiert. Die KJP-Vollversorgung wurde im Österreichischen Strukturplan Gesundheit (ÖSG) verankert und teilumgesetzt. KJPKassenstellen wurden geschaffen, und Vertreter der KJP tragen als Mitglieder wichtiger Gremien des Bundesministeriums für Gesundheit und Frauen (BMGF) zur Verbesserung der psychosozialen Gesundheit der PatientInnen bei. Im Folgenden werden einige Daten zum Ist-Stand und Soll-Stand der Versorgung dargestellt und diskutiert.

Zukunftsziel ist neben der Fachentwicklung der weitere differenzierte KJP-Versorgungsausbau, um möglichst vielen Kindern und Jugendlichen mit seelischen Erkrankungen eine Behandlung im öffentlichen Gesundheitssystem zu ermöglichen. Das Erreichen der Vollversorgung im Kernbereich und die Errichtung spezialisierter Versorgungsangebote bleiben Visionen.

\section{Die KJP-Ärzteausbildung}

Die Entwicklung der fachärztlichen Ausbildung hatte ihren Niederschlag in der neuen Ärzteausbildungsordnung, in welcher zuletzt im Juni 2016 die psychotherapeutische Expertise als zentraler Teil der Facharztausbildung im Rasterzeugnis integriert wurde. Es ist damit in den letzten 10 Jahren gelungen, das Sonderfach an ein internationales Niveau der Ausbildung heranzuführen. Der Titel: „Facharzt für Kinder- und Jugendpsychiatrie und psychotherapeutische Medizin“ steht für die aktuelle Facherweiterung.

Das Fach KJP entwickelte sich historisch aus den „Mutterfächern“ Pädiatrie, Psychiatrie und Neurologie, wobei FachärztInnen nach ihrer Facharztausbildung im Rahmen einer drei Jahre dauernden $\mathrm{Zu}$ satzausbildung das Additivfach „Kinderjugendneuropsychiatrie“ erlangen konnten. Mit der Entstehung des eigenständigen Sonderfaches KJP wurde die im Zusatzfach abgebildete neurologische Expertise und Versorgung der Pädiatrie zugeordnet, die fachärztliche Versorgung seelisch kranker Kinder- und Jugendlicher obliegt formal ab 2007 dem neuen Fach [3].

Seit 1985 war die wissenschaftliche Gesellschaft ÖGKJP (vormals ÖGKJNP) etabliert und konnte mit großer Erfahrung die Aufgaben der Ärzteausbildung durchführen. Sie wurde gemeinsam mit der ÖÄK, welche im übertragenen Wirkungsbereich für die Facharztausbildung verantwortlich ist, vom BMGF mit der Erarbeitung eines neuen, fachzentrierten Rasterzeugnisses betraut.

Im Rahmen der Überarbeitung der Ärzteausbildungsordnung (ÄAO) 2006 erreichte das Fach ein eigenes Rasterzeugnis, die ersten Fachärzte im Sonderfach KJP schlossen ihre Ausbildung 2007 ab.

Da in der ärztlichen Tätigkeit eine psychotherapeutische Expertise unabdingbar ist, hatten sich seinerzeit fast alle KollegInnen entschlossen, ihre Kenntnisse und Fertigkeiten um eine privat finanzierte Psychotherapieausbildung zu erweitern. Im Zuge der Novelle der ÄAO 2015 wurde in unserer Sonderfachausbil- dung die psychotherapeutische Dimension integriert und zur Umsetzung gebracht.

Laut Bundesgesetzblatt umfasst die Fachausbildung „die Prävention, Diagnostik, Behandlung einschließlich psychotherapeutischer Medizin und Rehabilitation von im Kindes- und Jugendlichenalters auftretenden psychischen Krankheiten, Störungen und Verhaltensauffälligkeiten einschließlich der psychiatrischen Behandlung von entwicklungsbedingten psychischen Erkrankungen sowie die fachspezifische Begutachtung" (BMGF 2015).

Auch die erste Grundsteinlegung zur spezialisierten Entwicklung unseres Faches ist gelungen. Ein Weiterbildungsangebot zum Erwerb der Zertifizierung „Kinder- und Jugendpsychiatrische Gutachten“ konnte ausgearbeitet und umgesetzt werden.

\section{Die KJP-Versorgungsentwicklung}

Die Schaffung des Sonderfaches führte zu einer Verankerung im österreichischen Strukturplan Gesundheit mit dem Ziel eines flächendeckenden Aufbaus von öffentlichen Versorgungsstrukturen für seelisch kranke Kinder und Jugendliche. Dies war die zweite wesentliche Konsequenz, welche es uns möglich machte, in der Planung Zielgrößen zu erarbeiten, die KJP-Versorgung zu beobachten und Umsetzungen zu begleiten.

Die ärztliche KJP-Versorgung stand bis zur Gründung des Sonderfaches außerhalb jeglicher ordnender Planungen der öffentlichen Gesundheitsstrukturen. Persönlicher Schwerpunkt von Kollegen und föderale Rahmenbedingungen waren zentrale Gestaltungsgrößen.

Kollegen mit der Zusatzqualifikation Kinder- und Jugendneuropsychiatrie boten mehrheitlich ihre Expertise im Rahmen ihres Mutterfaches Pädiatrie, Neurologie oder Psychiatrie an. Die nichtärztlichen Behandler (allied health professions) waren groß an der Zahl und trugen (und tragen) den größten Teil der extramuralen Versorgungsarbeit. Auch diesbezüglich gibt es keinerlei Planungsgrößen. Wir haben auf Basis der historischen Entwicklung unterschiedlich gewachsene Versorgungsangebote in einzelnen Regionen. Das Netzwerk der Behandlung unserer Patienten wird im Artikel von Fliedl et al. [4] (dieses Heft) dargelegt.

Dieser Artikel hat seinen Schwerpunkt in der Beschreibung der KJP-Versorgungsentwicklung des ärztlichen Kernbereiches. Beschrieben werden öffentliche KJP-Abteilungen, KJP-Ambulatorien und Ambulanzen sowie KJP-Kassenordinationen, somit jene Angebote, welche strukturell eine fachärztliche Anwesenheit verankert haben.

\section{Der intramurale Bereich}

Seit 2008 ist das Fach und die Versorgungsentwicklung in allen Regierungspapieren als Ziel erwähnt, ein Handlungsbedarf steht außer Streit und Bemühungen 
Abb. 1 Bettenmessziffern 2015

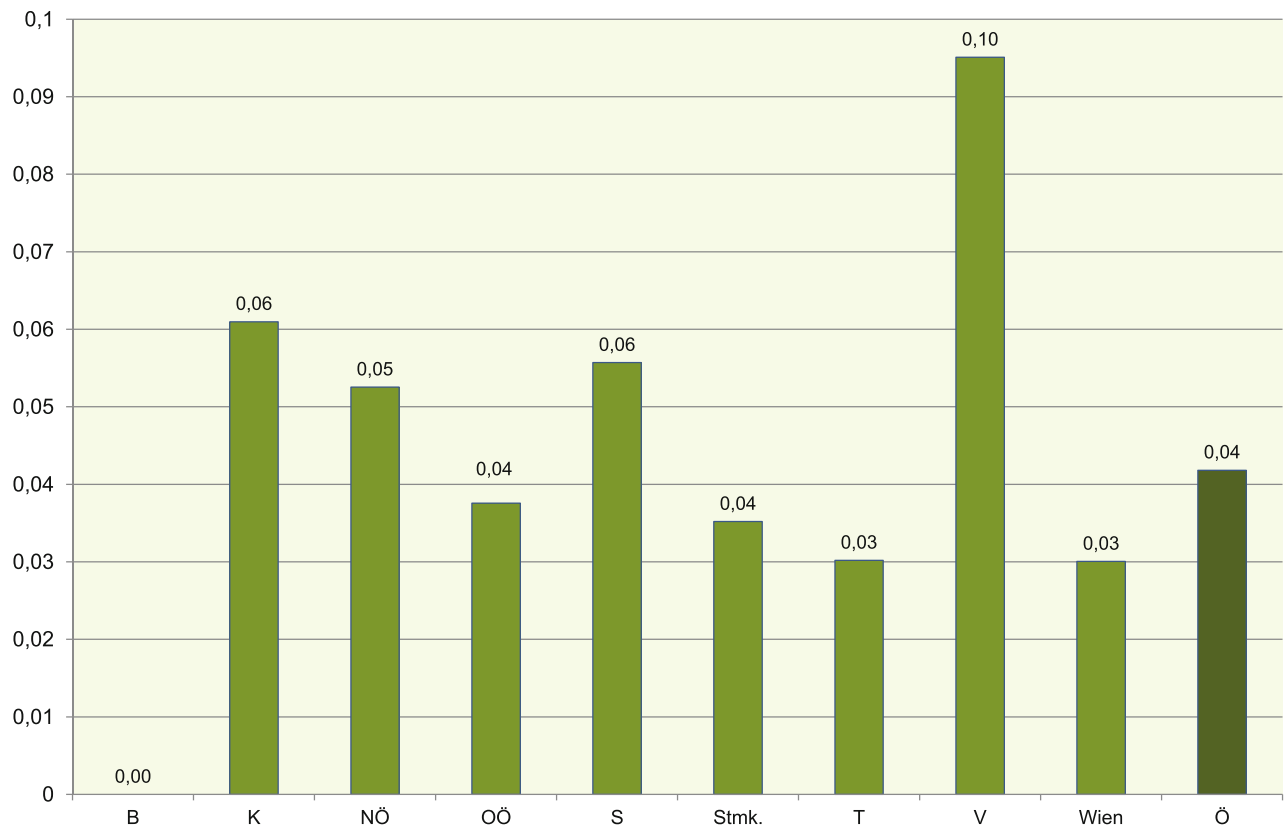

werden allerorts bekundet. Viele Jahre wurden Gespräche zur nachhaltigen Verbesserung der Gesundheit von Kindern und Jugendlichen geführt, wobei das ehemalige Bundesministerium für Gesundheit, nunmehr Bundesministerium für Gesundheit und Frauen (BMGF), mit insgesamt 180 Expertinnen und Experten aus den verschiedensten Bereichen der Kinderund Jugendgesundheit den Kindergesundheits-Dialog geführt und eine Kindergesundheitsstrategie erarbeitet hat [5].

Die Planung der öffentlichen Krankenbehandlung im intramuralen Bereich obliegt im Auftrag des Gesundheitsministeriums der Gesundheit Österreich GSMBH (GÖG). Ein „Österreichischer Strukturplan Gesundheit - ÖSG“ wird regelmäßig erarbeitet, welcher dann in den Bundesländern als „Regionaler Strukturplan Gesundheit - RSG“ zur Anwendung gebracht werden soll.

Ab 2008 wurden Vertreter des Sonderfaches KJP zu einer Vielzahl von fachzentrierten Verhandlungen geladen. Prävalenz und internationale Versorgungszahlen fanden Eingang in diese Gespräche. In den ÖSGVerhandlungen hat man sich auf eine Zielgröße der Bettenmessziffer (BMZ = 1 intramuraler Behandlungsplatz pro $1000 \mathrm{EW}$ inkl. tagesklinischer Plätze) von 0,08-0,13 geeinigt (Abb. 1).

Die 9 Landesfonds melden jährlich ihre Bettenzahlen (inklusive tagesklinischer Behandlungsplätze) an das BMGF. In Abb. 2 überblicken wir die Entwicklung der Betten im Zeitraum 2004-2015.

Zusammenfassend kann für den intramuralen Bereich festgehalten werden, dass wir erst die Hälfte der dringlich erforderlichen KJP-Betten in der Grundversorgung zur Verfügung haben. Es wurde in den letzten 10 Jahren ein Zuwachs von 56 Behandlungsplätzen erreicht, der derzeitige Bettenausbau entspricht einer bundesweiten BMZ von 0,05. Zwischen den ein- zelnen Bundesländern bestehen große Unterschiede, nur Vorarlberg hat seit 2015 intramural die geplante Vollversorgung erreicht.

\section{Die extramurale Versorgung}

Die extramurale Versorgung steht traditionsgemäß auf zwei Säulen, den niedergelassenen FachärztInnen und den Ambulatorien. Es war den Fachvertretern der KJP wichtig, dass dies auch für Kinder und Jugendliche mit seelischen Erkrankungen gelten sollte. Viele Widerstände waren zu überwinden.

Laut österreichischer Sozialversicherungsträger, Strategie psychischer Gesundheit 2012 (3), ist es das erklärte Ziel der Versicherungsträger, eine niederschwellige und flächendeckende Grundversorgung für Kinder zu gewährleisten. „In der ambulanten Behandlung bekennt sich Österreich in der Kinder- und Jugendlichenpsychiatrie zum bestehenden „dualen System“ mit niedergelassenen FachärztInnen und mit „Integrierten Fachambulatorien " mit multiprofessionellen Teams" (Strategie psychische Gesundheit der Sozialversicherungsträger 2012 S. 20) [5].

Unser erklärtes Ziel ist es, das rezente Zitat des Altrektors der Medizinischen Universität Wien Prof. W. Schütz mittelfristig als überholt betrachten zu dürfen, der 2017 (Schütz W, 2017, 68) schreibt: „Über die ambulante Versorgung im Fach der Kinder- und Jugendpsychiatrie wäre überhaupt der Mantel des Schweigens zu breiten: $10 \%$ der Kinder und Jugendlichen würden psychotherapeutische Hilfe benötigen, unter $1 \%$ erhalten sie [6]. “

\section{Versorgung durch KJP-Ambulatorien, Tageskliniken und Ambulanzen}

Laut ÖSG sollte zur Versorgung von Kindern und Jugendlichen mit komplexen Störungsbildern ein KJP- 

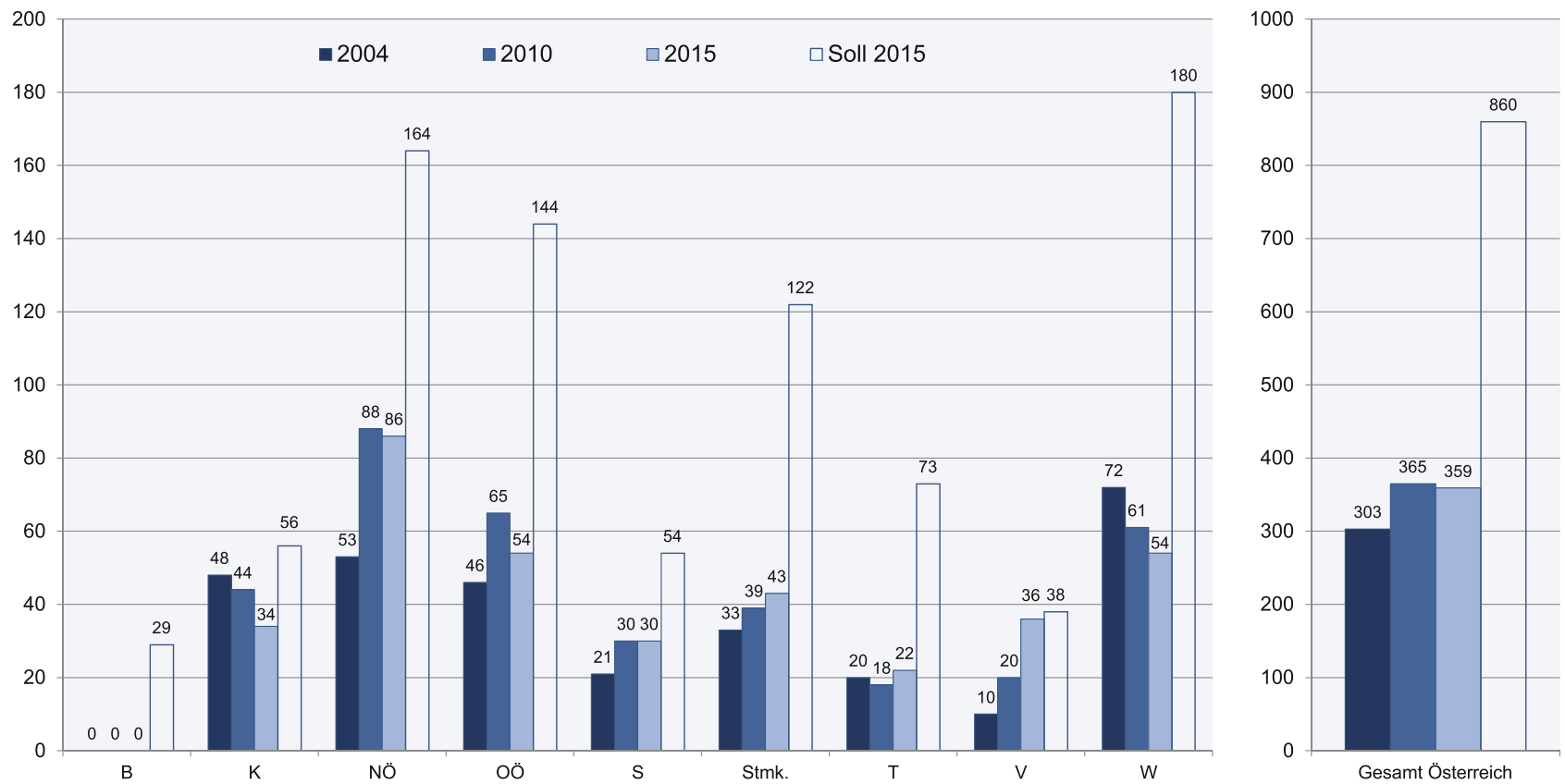

Abb. 2 Bettenmessziffern 2004-2015 sowie Sollwert 2015

Ambulatorium bzw. 1 Ambulanz auf 250.000 EW errichtet werden.

In den letzten 10 Jahren ist es nicht gelungen, bundesweite, bindende Strukturkriterien eines KJP-Ambulatoriums im ÖSG so zu verankern, sodass diese von allen Entscheidungsträgern im Konsens umgesetzt werden könnten.

Viele Abteilungen haben auch eine Ambulanz, gleichzeitig gibt es eigenständige Ambulatorien, die hauptsächlich von privaten Trägern betrieben werden. Da die Einrichtungen von ihren Ressourcen und Aufgabenstellung und damit in ihrer Versorgungsrelevanz sehr unterschiedlich sind, ist es derzeit nicht möglich, entsprechende Versorgungszahlen von Ambulatorien aufzulisten.

In manchen Bundesländern wurden im letzten Jahr dislozierte Tageskliniken von KJPP-Abteilungen mit einer angeschlossenen KJPP-Ambulanz errichtet. Diese geben den Familien als wohnortnahe, höherschwellige Angebote die Möglichkeit einer verbesserten, lokal vernetzten Behandlung und poststationären Reintegration. Bei Bedarf ist die vollstationäre Aufnahme ohne Schwelle möglich. Die tagesklinischen Plätze an den KJPP-Abteilungen sind als stationäre Behandlungsplätze in Abschn. 2.1 mitberücksichtigt, die dislozierten Behandlungsplätze waren 2015 noch nicht errichtet.

Ergänzend sind Stand-alone Tageskliniken anzuführen. Da diese von privaten Trägern geführt werden, sind wir derzeit nicht im Besitz von zusammengeführten Daten.

\section{Die kassenärztliche Versorgung}

Die Möglichkeit zur fachärztlichen Versorgung durch niedergelassene Kassenfachärzte war eine weitere wesentliche Auswirkung der Sonderfachetablierung. Die damit verknüpfte Gründung einer eigenen Fachgruppe als politische Vertretung machte diese Behandlungsoption in direkten Verhandlungen mit den Gebietskrankenkassen in bisher 7 Bundesländern möglich.

Eine wesentliche Säule der Versorgung von Patienten in unserem Land ist die Behandlung durch niedergelassene FachärztInnen (Kassenärzte). Im Sinne der Gleichbehandlung aller PatientInnen war es in der politischen Arbeit ein besonders Anliegen, auch Kindern und Jugendlichen mit seelischen Erkrankungen im öffentlichen Gesundheitssystem die Möglichkeit der Behandlung bei ihrem „Facharzt des Vertrauens“ zu ermöglichen.

Die Verhandlungen zur Etablierung von Kassenstellen werden in unserem Land von den Gebietskrankenkassen und den Länderärztekammern geführt. Mit der Schaffung des eigenen Sonderfaches wurden in den Länderärztekammern politische Vertreter der Fachgruppe KJP gewählt. Diese konnten fachzentrierte Anliegen zur Niederlassung in den Gremien einbringen. Erst durch die Konstituierung der eigenen Fachgruppe war das Sonderfach KJP in diesen Strukturen vertreten und leistete in diese Form ihren Beitrag zur Entwicklung von neuen Versorgungsstrukturen.

Die angeführten, föderalen Gremien entscheiden gemeinsam in regelmäßigen Stellenplangesprächen über die Versorgungsentwicklung durch niedergelassene KassenärztInnen. Für die Schaffung eines KJPKassenvertrages war die Erarbeitung eines Leistungskataloges, der die Notwendigkeiten der KJP-Arbeitsweise abbildet, erforderlich. Da sich die Tätigkeit von FachärztInnen für KJP als Teil eines multimoda- 

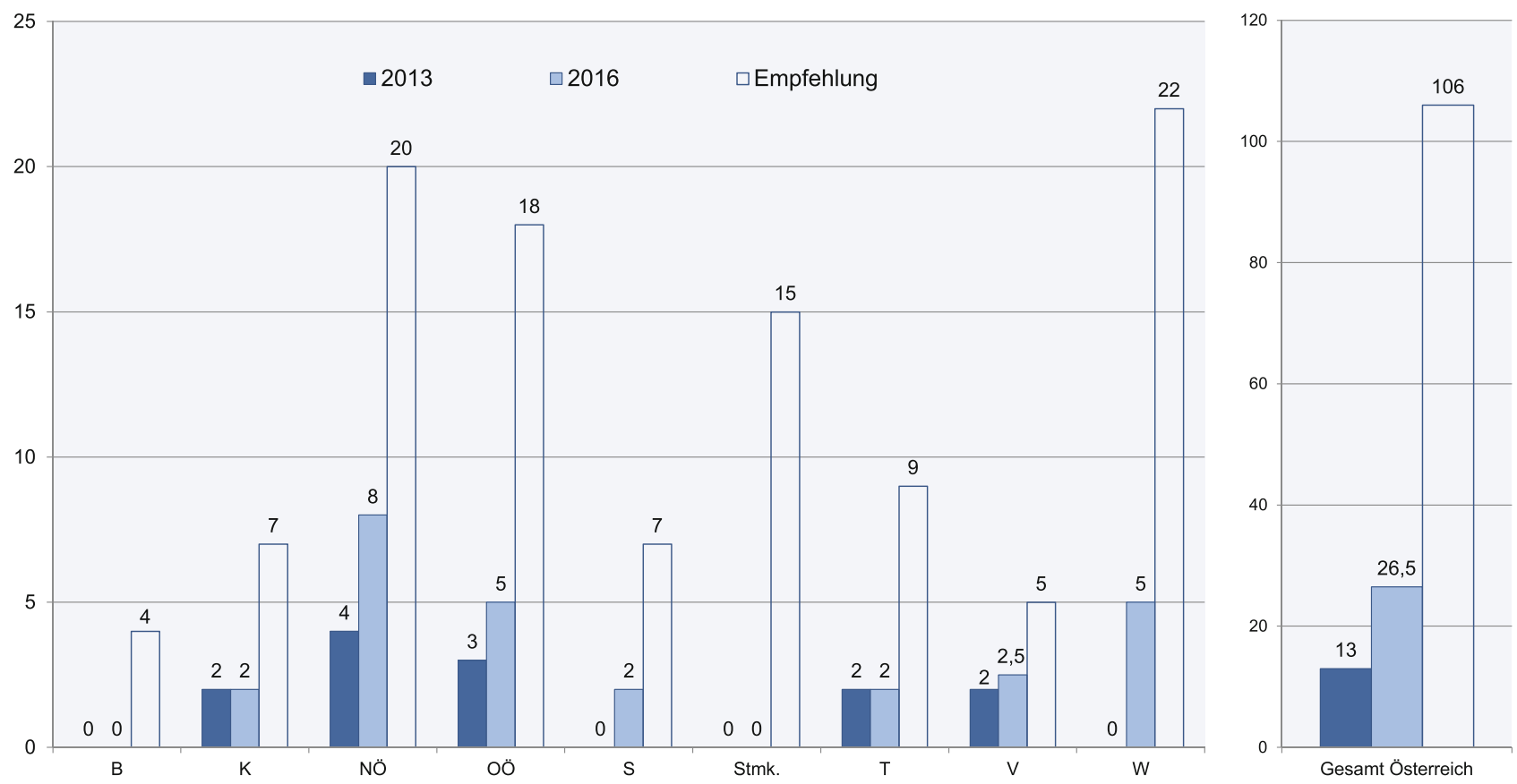

Abb. 3 Entwicklung der kassenärztlichen Ordinationen

len Netzwerkes wesentlich von der herkömmlichen Arbeit von KollegInnen anderer Fachgruppen unterscheidet, begaben wir uns auf einen langen Weg des gemeinsamen Abwägens und Verhandelns.

Nach einer Projektierungs- und Evaluierungsphase von 2005-2012 hat die NÖ-Fachgruppe mit der NÖGKK einen ersten, fachzentrierten Leistungskatalog fertiggestellt und 2012 mit 4 KJP-Kassenstellen begonnen. In einigen Bundesländern erarbeiteten die KollegInnen zeitnah mit den Gebietskrankenkassen (GKK's) ähnliche Verträge. Die Honorierung des Patientengesprächs und die strukturell verankerte Kooperation mit anderen Versorgern war neben der primären ärztlichen Tätigkeit ein wichtiger Bestandteil der Verhandlungsziele. Diese Arbeitsbereiche bilden sich in den verschiedenen KJP-Leistungskatalogen ab.

Die Verhandlungen mit den bundesweiten Kassen obliegen den Vertretern der ÖÄK. Unter Zuhilfenahme der Basisarbeit aus NÖ wurde auch hier ein Leistungskatalog erstellt und die Kassenverträge umgesetzt. Primär konnte mit der VAEB ein Vertrag abgeschlossen werden, die anderen Träger der „kleinen Kassen“ folgten innerhalb eines Jahres.

In der Abb. 3 wird die Entwicklung der kassenärztlichen Ordinationen länderspezifisch dargestellt (Abb. 3).

In Wien haben 2017 vier Einzelordinationen und eine Gruppenpraxis ihre Arbeit aufgenommen, in Salzburg ist 1 Kassenordination in Betrieb, die 2. Kassenstelle konnte noch nicht besetzt werden. Bei einer Zielgröße von 1 KJP-Kassenstelle auf 80.000 EW (einem untersten Wert der Versorgung in den deutschsprachigen Nachbarländern) - haben wir dzt. ein Viertel der notwendigen Kassenstellen besetzt. Be- dauerlicherweise haben sich die GKK's von Steiermark und Burgenland weiterhin von der kassenärztlichen Versorgung distanziert, wenngleich ein Bekenntnis der Versicherungsträger existiert.

\section{Hoch spezialisierte Versorgungsbereiche}

Die Vertreter des Sonderfaches sind sich bewusst, dass viele Versorgungsbereiche wie Angebote für die Behandlung von Essstörungen, Suchterkrankungen, der forensische Bereich und der Transition ins junge Erwachsenenalter mehrheitlich in der Realität und den politischen Planungen, die derzeit vorliegen, noch gar nicht berücksichtigt sind.

Diese konnten noch nicht entwickelt werden, da der Kernbereich derzeit noch deutlich von einer Vollversorgung entfernt ist. Große Anstrengungen aller Entscheidungsträger werden erforderlich sein, um uns an internationale Versorgungsgrößen anzunähern. Das neue Sonderfach KJP hat mit der Umsetzung der Kassenplanstellen in den letzten 10 Jahren in fast allen öffentlichen Versorgungsstrukturen seinen Platz gefunden.

Die Rehabilitation steht in der Versorgungsplanung vor einer Umsetzung. Es wurden in den 4 Versorgungszonen 109 Rehabilitationsplätze genehmigt und auch ausgeschrieben, 3 Regionen sind zum gegenwärtigen Zeitpunkt besetzt, wobei nur 42 Plätze für den Kernbereich KJP für ganz Österreich vorgesehen sind (Tab. 1).

Der derzeitige Rehabilitationsplan [7] kann aus unserer Sicht als Beginn eines Entwicklungsprozesses gesehen werden. Die Zukunft wird zeigen, inwieweit 
Tab. 1 Bettenbedarffür Kinder und Jugendliche (0-18 Jahre) im Jahr 2020 (Soll) für KJP und Pädiatrische Psychosomatik (ESP) nach Versorgungszonen

\begin{tabular}{|l|l|}
\hline Versorgungszone & Soll 2020 (ESP, KJP) \\
\hline Ost & 46 \\
\hline Süd & 24 \\
\hline Nord & 24 \\
\hline West & 15 \\
\hline Österreich (gesamt) & 109 \\
\hline $\begin{array}{l}\text { KJPErkrankungen in der Kinder- und Jugendpsychiatrie, ESPEntwicklungs- } \\
\text { und Sozialpädiatrie sowie pädiatrische Psychosomatik }\end{array}$
\end{tabular}

ergänzend wohnortnahe, familienzentrierte, kleinere Rehabilitationsangebote erforderlich sein werden.

Zusammenfassend können wir uns der Diktion der österreichischen Sozialversicherungsträger anschließen: „In der Versorgung von psychisch erkrankten Kindern und Jugendlichen besteht derzeit ein noch nicht ausreichend gedeckter Bedarf im ambulanten Bereich und regional unterschiedlich auch im stationären Bereich. Ambulanter und stationärer Bereich müssen in der Kinder- und Jugendlichenpsychiatrie in einem besonderen Ausmaß gut vernetzt entwickelt werden " [5].

Die nächsten Jahre werden zeigen, wie die Versorgungsentwicklung vorangetrieben wird. Da die FachärztInnenausbildung hauptsächlich in den Krankenanstalten stattfindet, fehlen durch mangelnden Ausbau der bettenführenden Abteilungen die dringend notwendigen Ausbildungsplätze.

\section{Die Berufsgruppe der FachärztInnen im Sonder- fach Kinder- und Jugendpsychiatrie}

Der Beginn des Sonderfaches war gleichbedeutend mit der Etablierung der eigenen Fachgruppe in den Länderärztekammern und in weiterer Folge in der ÖÄK. Die vielschichtigen Aufgaben der politischen Vertretung sollten in enger Kooperation mit allen Vertretern der Versorgungssegmente erfolgen, um alle Ressourcen optimal nutzen zu können. Der FachärztInnenmangel führte häufig zu Spannungen innerhalb der Fachgruppe und den Versorgungssegmenten. Die Vielzahl von unversorgten Patienten zwingt in der ärztlichen Arbeit zur täglichen Triagierung, eine leitliniengerechte Behandlung ist dabei für viele PatientInnen kaum oder zu spät möglich. Der massive Ressourcenmangel bedingt ein Gefühl der Hilflosigkeit und Überforderung, ein weiterer Aspekt für alle politisch tätigen KollegInnen, die Verbesserung der Versorgung unbeirrt einzufordern.

Um das Fach in die Niederlassung führen zu können, war klar, dass vermehrt KollegInnen ausgebildet werden mussten. Die FachärtzInnenentwicklung ist ein zentraler Faktor für die Umsetzung des Versorgungsauftrages in allen Bereichen war. Aus der Versorgungsplanung der Kernbereiche (Abb. 4) wurde ein Fachärztebedarf von 300-350 FachärztInnen abgeleitet, die Entscheidungsträger reagierten wie folgt:

Dem seit längerer Zeit bestehenden Mangel an Fachärzten im Sonderfach Kinder- und Jugendpsychiatrie

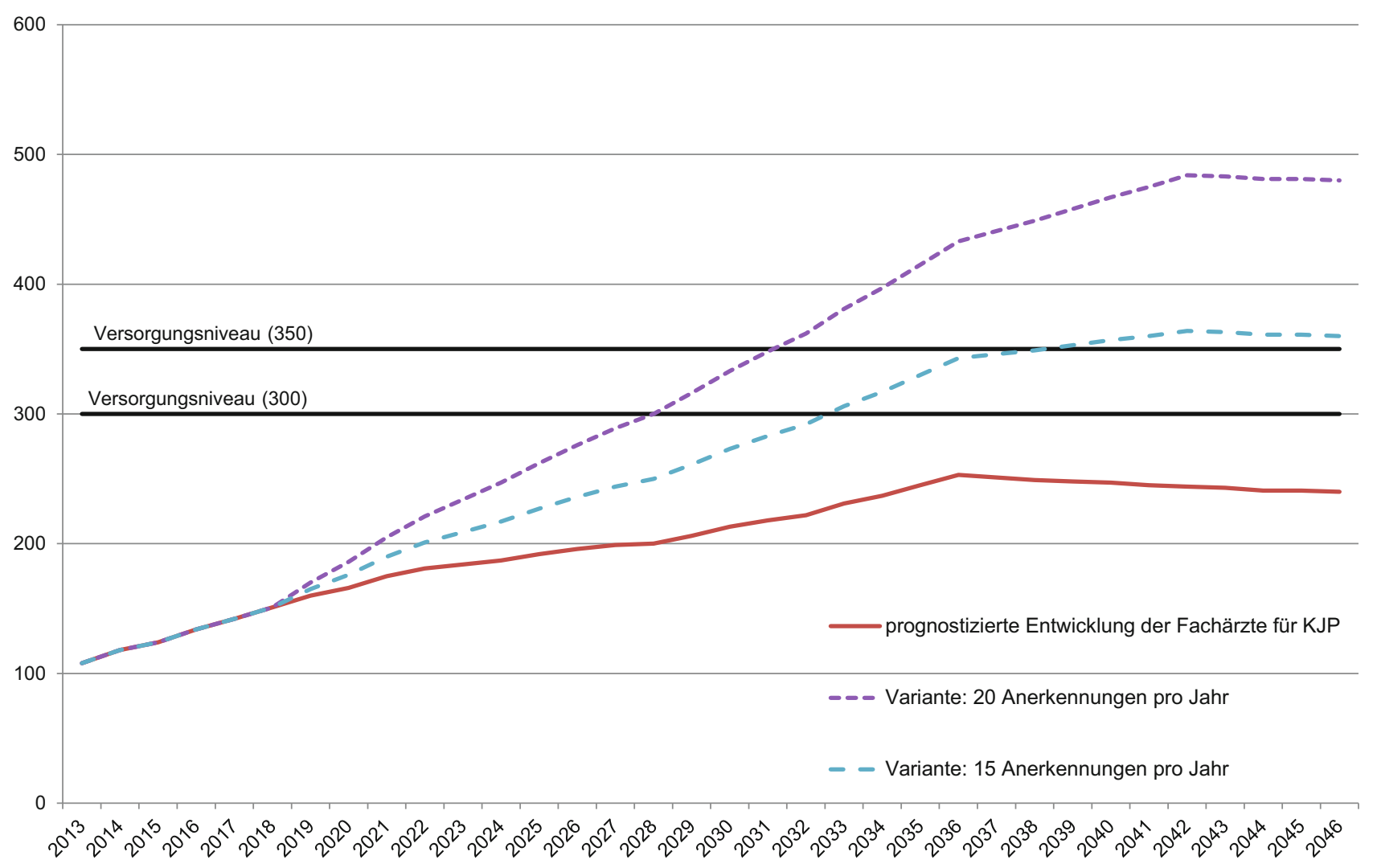

Abb. 4 Prognose der Facharztentwicklung mit 2013 (alte ÄAO) 
soll durch Nutzung der mit der 12. Ärztegesetz-Novelle, BGBl. I Nr. 62/2009, in \$ 10 Abs. 4 ÄrzteG 1998 geschaffenen Verordnungsermächtigung insoweit begegnet werden, als dieses Sonderfach zeitlich bis zum 30. Juni 2016 zum Mangelfach erklärt wird. [6]. https://www. ris.bka.gv.at/Dokumente/Begut/.../COO_2026_100_2_ 591937.html.

Durch diese Regelung wurde es möglich, die Ausbildungsstellen pro Abteilung um 1 Stelle zu vermehren.

Die Wirksamkeit dieser politischen Intervention wurde 2013 von Seite der Ärztekammern mit Zahlen überprüft.

Historisch bedingt war die Mehrheit der KollegInnen in den Mutterfächern verortet. Die KJP-Abteilungen mussten erst aufgebaut, Ausbildungsstellen geschaffen oder ausgebaut werden. Auch extramural hatten wir bis 2012 kaum KollegInnen in der Niederlassung.

2013 versuchten die Standesvertreter der Länderärztekammern mit einer internen Befragung der KollegInnen, einen Überblick zu bekommen, wie viele der damals 161 KollegInnen im KJP-Kernbereich relevante Versorgung leisteten. Bundesweit arbeiteten damals nach einer Länderbefragung erst 98 KollegInnen im Kernbereich (Bedarf 300-350). Die übrigen KollegInnen waren weiter in ihren Mutterfächern versorgungswirksam. Neben der Ist-Standerhebung wurde auch eine Prognose der ärztlichen Personalentwicklung erstellt. Diese zeigte, dass wir mit der damaligen Mangelfachregelung auch zukünftig keine Vollversorgung erreichen würden. Ein zusätzlicher politischer Schritt zur Erweiterung der Ausbildungsplätze wurde notwendig.

Im Rahmen der ÄAO 2016 können pro Abteilung 2 weitere Ausbildungsstellen beantragt werden. Im Gesetzestext heißt es wie folgt: „Übergangsbestimmung für Mangelfachregelungen: $\$$ 37. (1) Bis 31 . Mai 2021 ist für die Ausbildung im Sonderfach Kinder- und Jugendpsychiatrie sowie im Sonderfach Psychiatrie und Psychotherapeutische Medizin im Rahmen der Anerkennung einer Ausbildungsstätte bei der Festsetzung von mehr als einer Ausbildungsstelle der Ausbildungsverantwortliche auf die Zahl der zu beschäftigenden weiteren Fachärztinnen/Fachärzte bis zu einem Höchstmaß von drei weiteren Ausbildungsstellen anzurechnen, so dass für die Bewilligung der ersten vier Ausbildungsstellen die Anleitung und Aufsicht der Turnusärztinnen/Turnusärzte durch zwei Fachärztinnen/Fachärzte als ausreichend angesehen werden. Für jede weitere Ausbildungsstelle ist eine weitere Fachärztin/ein weiterer Facharzt in Vollzeitbeschäftigung (oder auch mehrere teilzeitbeschäftigte Fachärztinnen/Fachärzte im Ausmaß einer Vollzeitbeschäftigung) zu beschäftigen. (2) Eine Ausbildung in dem im Absatz 1 genannten Sonderfach, die bis 31. Mai 2021 begonnen worden ist, darf unter Anwendung von Abs. 1 auch über diesen Zeitpunkt hinaus fortgesetzt und abgeschlossen werden." [8] (https://www.ris.bka.gv.

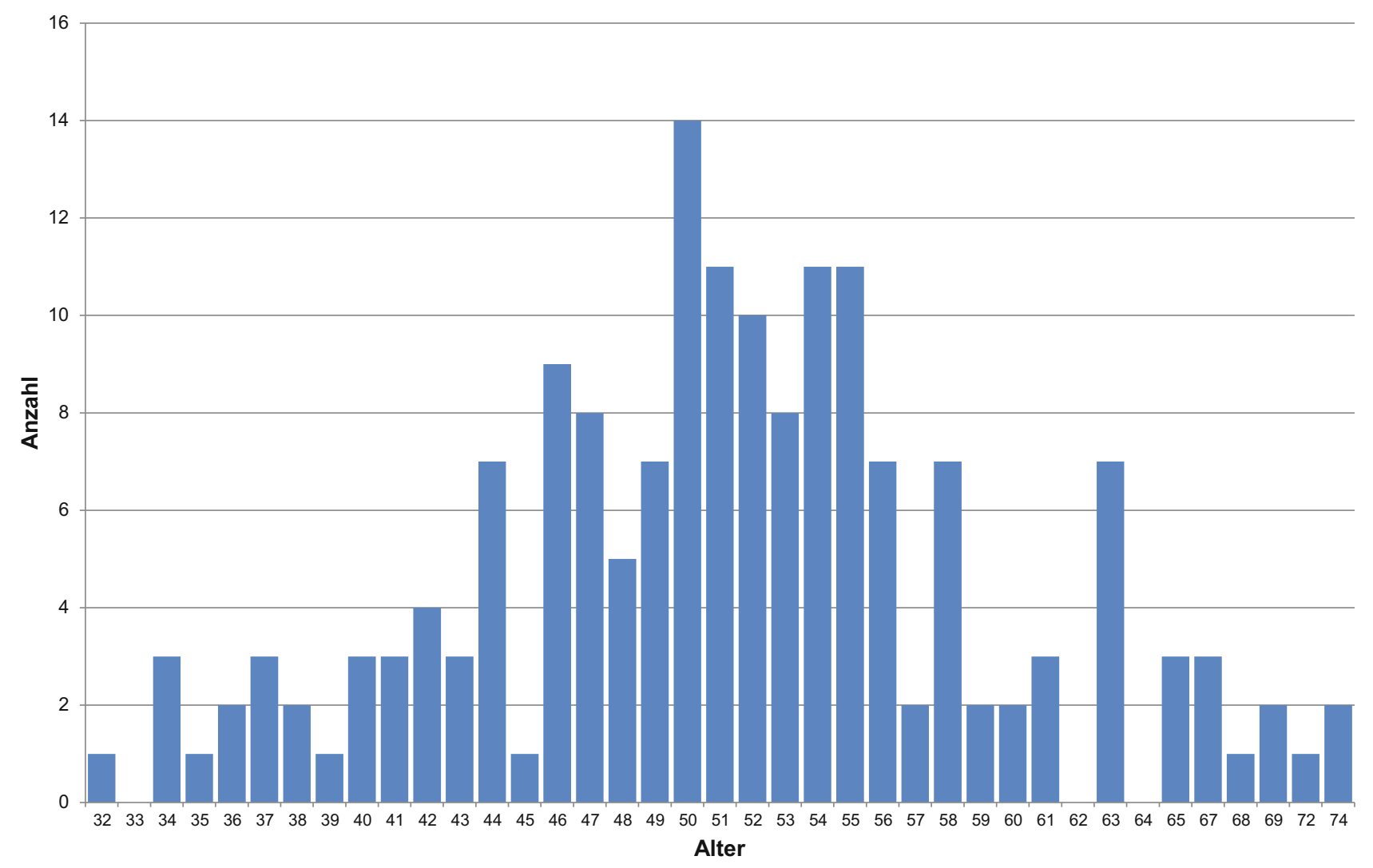

Abb. 5 Altersverteilung Fachärzte Österreich Stand 2017 


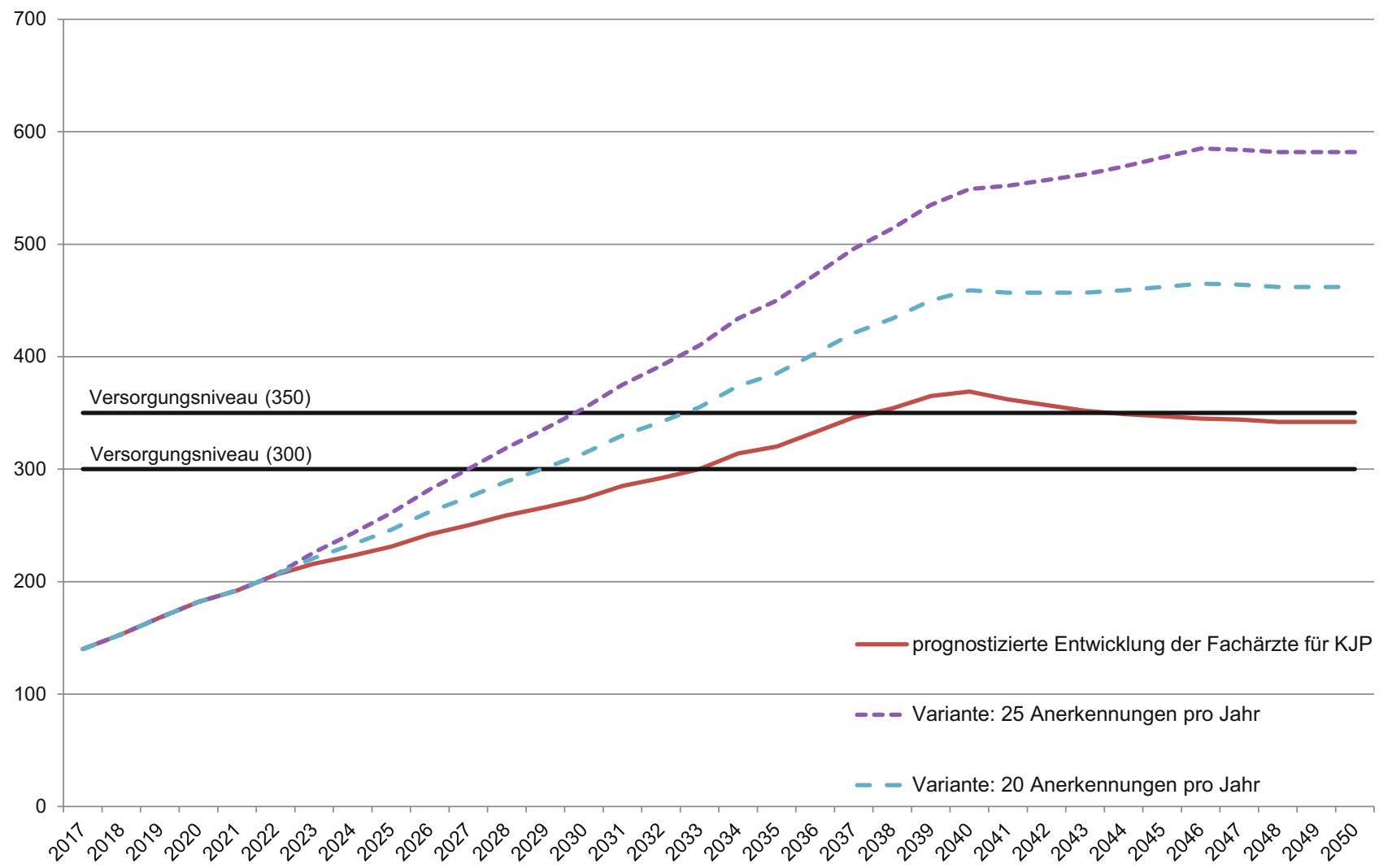

Abb. 6 Prognose der Facharztentwicklung mit 2017 (neue ÄAO)

at/GeltendeFassung.wxe?Abfrage=Bundesnormen\& Gesetzesnummer=20009186).

Um die langfristige Wirksamkeit der Gesetzesänderung zu beobachten, haben wir in der ÖÄK 2017 eine aktuelle KJP-Ärzteentwicklungsstatistik als IST-Stand für das Fach KJP ausgearbeitet. Die Altersverteilung jener KollegInnen, welche unserem Fach angehören, findet sich in Abb. 5.

Wir sind davon ausgegangen, dass jene KollegInnen, welche primär im Sonderfach ausgebildet werden, mehrheitlich in den neu geschaffenen KJP-Bereichen tätig sein werden. Mit dem Ausbau unterschiedlicher Versorgungsbereiche sind jetzt in vielen Bundesländern entsprechende KJP-Arbeitsplätze geschaffen worden.

Die Berechnung 2017 ergibt folgenden Kurvenverlauf, für die Versorgungsprognose wurde ein Pensionsantrittsalter von 65 Jahren angenommen.

Als Jahresmittelwerte der neuen Anerkennungen (= erfolgreiche Facharztprüfungen) müssen wir inzwischen 15 ansetzen. Dies stellt auch die Hauptvariante dar. Alternativ wurde ab dem Zeitraum +5 Jahre (also ab 2023) ein Durchschnittswerte von 20 bzw. 25 angesetzt. Damit ist dieser angenommene Zuwachs $(15 \rightarrow$ 20: $+33 \%$ und $15 \rightarrow 25:+66 \%$ ) geringer angesetzt als im letzten Modell $(10 \rightarrow 15:+50 \%$ und $10 \rightarrow 20$ : $+100 \%)$. In diesem Szenario durchschlägt sogar die Hauptvariante etwa 2034 den höheren Versorgungsgrad von 350, während sie im letzten Modell den niedrigeren Grad von 300 nicht erreicht hat. (Mag. A. Sinabell/ÖÄK) (Abb. 6).

Aus politischer Sicht ergibt sich aus der neuen Ausbildungsordnung eine bessere Prognose in der Fachgruppenentwicklung. Es lässt erwarten, dass wir mit der derzeitigen Ausbildungsregelung ein unteres Versorgungsniveau von ca. 300 KollegInnen im Kernbereich mit dem Jahr 2033 erreichen werden. Mit März 2017 werden in der Standesliste der ÖÄK 234 KPJFachärztInnen geführt, 91 KollegInnen sind nicht im KJP-Kernbereich versorgungswirksam, 143 schon. Es ist geplant, in fünf Jahren eine weitere Evaluierung durchzuführen.

Die gesetzlichen Grundlagen können lediglich den Rahmen schaffen, die tatsächliche Umsetzung obliegt den Bundesländern. Jeder Träger ist aufgerufen, ausreichend KollegInnen in Ausbildung zu nehmen, um für die Zukunft vorzubauen.

\section{Conclusio}

Die Notwendigkeit einer KJP-Versorgung steht außer Streit, alle Entscheidungsträger bekennen sich zur KJP-Mangelversorgung, in der Umsetzung sind Teilerfolge zu verzeichnen, die gesetzlichen Rahmenbedingungen zur Fachentwicklung wurden geschaffen.

Es bleibt zu hoffen, dass die nächste Generation von FachärztInnen für KJPP unseren PatientInnen in naher Zukunft leitliniengerechte fachärztliche Versor- 
gung anbieten kann. Die Verantwortung obliegt den Entscheidungsträgern.

Danksagung Wir danken Herrn Mag. A. Sinabell von der ÖAK für die Zurverfügungstellung der relevanten Zahlen und die Berechnungen der Ärztestromanalyse sowie Herrn Mag. Michael Zeiler für die graphische Optimierung.

Interessenkonflikt C. Hartl und A. Karwautz geben an, dass kein Interessenkonflikt besteht.

Open Access Dieser Artikel wird unter der Creative Commons Namensnennung 4.0 International Lizenz (http:// creativecommons.org/licenses/by/4.0/deed.de) veröffentlicht, welche die Nutzung, Vervielfältigung, Bearbeitung, Verbreitung und Wiedergabe in jeglichem Medium und Format erlaubt, sofern Sie den/die ursprünglichen Autor(en) und die Quelle ordnungsgemäß nennen, einen Link zur Creative Commons Lizenz beifügen und angeben, ob Änderungen vorgenommen wurden.

\section{Literatur}

1. Thun-Hohenstein L. (Hrsg.) Kinder- und Jugendpsychiatrie in Österreich: Vom „Gestern“ zum „Morgen“. Wien: Krammer;2007.
2. WarnkeA, LehmkuhlG. Kinder-und Jugendpsychiatrie und Psychotherapie in Deutschland. Die Versorgung von psychisch kranken Kindern, Jugendlichen und ihren Familien. Stuttgart: Schattauer; 2011.

3. KarwautzA,Purtscher-PenzAK,HochgattererP,Kienbacher C, Board of the Austrian Society of Child and Adolescent Psychiatry. Child and adolescent psychiatry in Austria. Eur Child Adolesc Psychiatry. 2015;24(3):359-60. doi:10.1007/ s00787-014-0657-1.

4. Fliedl R, Sevecke K. Zur Psychodynamik von Organisation und Kooperation in der Kinder- und Jugendpsychiatrie. Neuropsychiatr (2017) 31. doi: 10.1007/s40211-017-0247-

5. Hauptverband 2012: http://www.hauptverband.at/cds content $/$ load? contentid $=10008.564642 \&$ version $=139118$ 4577.Zugegriffen: 01.07.2017

6. Schütz W. Eintritt nur nach Aufruf: Warum Österreich die Ärzte ausgehen: elf Übel, elfFakten. Wien:Manz; 2017.

7. Gyimesi M, Fülöp G, Ivansits S, Pochobradsky E, Stoppacher A, Kawalirek S, Maksimovic A. Rehabilitationsplan 2016. Wien: GesundheitÖsterreich; 2016.

8. BMGF-Gesetzestext ÄAO 2015: https://www.ris.bka. gv.at/GeltendeFassung.wxe?Abfrage=Bundesnormen\& Gesetzesnummer=20009186.Zugegriffen:01.07.2017 